\title{
Monolayer Graphene as a Saturable Absorber in a Mode-Locked Laser
}

\author{
Qiaoliang Bao ${ }^{1, \S}$, Han Zhang ${ }^{2, \S}$, Zhenhua $\mathrm{Ni}^{3}$, Yu Wang ${ }^{1}$, Lakshminarayana Polavarapu', Zexiang Shen ${ }^{3}$, \\ Qing-Hua $X u^{1}$, Dingyuan Tang ${ }^{2}(\bowtie)$, and Kian Ping $\operatorname{Loh}^{1}(\bowtie)$ \\ ${ }^{1}$ Department of Chemistry, National University of Singapore, 3 Science Drive 3, Singapore 117543, Singapore \\ ${ }^{2}$ School of Electrical and Electronic Engineering, Nanyang Technological University, Singapore 639798, Singapore \\ ${ }^{3}$ School of Physical and Mathematical Sciences, Nanyang Technological University, Singapore 637371, Singapore \\ $\S$ These authors contributed equally to this work. \\ Received: 16 August 2010 / Revised: 15 November 2010 / Accepted: 16 November 2010 \\ (C) Tsinghua University Press and Springer-Verlag Berlin Heidelberg 2010
}

\begin{abstract}
We demonstrate that the intrinsic properties of monolayer graphene allow it to act as a more effective saturable absorber for mode-locking fiber lasers when compared to multilayer graphene. The absorption of monolayer graphene can be saturated at lower excitation intensity compared to multilayer graphene, graphene with wrinkle-like defects, or functionalized graphene. Monolayer graphene has a remarkably large modulation depth of $65.9 \%$, whereas the modulation depth of multilayer graphene is greatly reduced due to nonsaturable absorption and scattering loss. Picosecond ultrafast laser pulses (1.23 ps) can be generated using monolayer graphene as a saturable absorber. Due to the ultrafast relaxation time, larger modulation depth and lower scattering loss of monolayer graphene, it performs better than multilayer graphene in terms of pulse shaping ability, pulse stability, and output energy.
\end{abstract}

\section{KEYWORDS}

Graphene, saturable absorber, laser, carrier dynamics, ultrafast photonics

\section{Introduction}

Graphene is an atomic layer of conjugated $\mathrm{sp}^{2}$ carbon atoms arranged in a two dimensional hexagonal lattice. Charge carriers in graphene move at ultrafast speed behaving like relativistic, massless Dirac particles [1, 2]. Most research attention thus far has been focused on the unique electronic structure of graphene. Comparatively less effort has been dedicated to investigate the photonic properties and applications of graphene, although graphene shows exciting potential in ultrafast photonics devices because of its ultrafast carrier dynamics [3, 4] and large, broadband optical absorption $(2.3 \%$ per layer $)[5,6]$. Previously, we have demonstrated the application of few-layer graphene [7-9] as well as graphene-polymer composites [10,11] as saturable absorbers in mode-locked lasers. Subsequently, another two groups also confirmed the mode-locking of lasers using chemically processed graphene film [12] and graphene-polymer composites [13] as saturable absorbers. In all these experiments, the saturable absorbers were multilayer graphene

Address correspondence to Dingyuan Tang, edytang@ntu.edu.sg; Kian Ping Loh, chmlohkp@nus.edu.sg 
films or graphene composites and the unambiguous demonstration of saturable absorption from a single atomic layer of graphene film has yet to be achieved. In principle, the excited carriers in pristine monolayer graphene are expected to show ultrafast decay (similar to graphite in tens of femtoseconds [14]) due to the zero gap in graphene. This therefore raises a fundamental question: Can the excited carriers in pristine monolayer graphene fill all the states in the conduction band or reach equilibrium under such a fast carrier relaxation rate? It must also be recognized that the optical and electrical properties of atomically thin graphene will be very sensitive to defects. The influence of sample quality and surface states on the saturation absorption properties has not yet been investigated.

In this work, in contrast to all previous studies that examined composite polymer or multilayer films involving graphene, we have painstakingly isolated a single atomic layer of graphene on an optical fiber and studied its nonlinear absorption properties. The performance is compared with monolayer graphene with surface defects, and monolayer graphene modified with functional groups, as well as multilayer graphene. We found that the threshold energy for saturation correlates with nonsaturable loss and scattering effects, all of which scale with the number of defects or thickness of the film. In addition, monolayer graphene is found to have faster carrier-carrier and carrier-phonon intraband scattering processes than multilayer graphene.

\section{Experimental}

Large area monolayer and multilayer graphene films were grown by chemical vapor deposition (CVD) on $\mathrm{Cu}$ [15] and $\mathrm{Ni}$ respectively. $\mathrm{Cu}$ and $\mathrm{Ni}$ [16] were then etched away in $\mathrm{FeCl}_{3}$ solution, followed by the transfer of the graphene films onto optical fibers or a quartz substrate, as described in our previous work [7]. Monolayer graphene was functionalized covalently with nitrobenzene by immersing the end face of a fiber pigtail in 4-nitrobenzenediazonium tetrafluoroborate (Sigma-Aldrich) solution $(0.01 \mathrm{mg} / \mathrm{mL})$ for $10 \mathrm{~min}$. The sample was then washed with deionized water. The Raman spectra and images were measured on a WITec CRM 200 Raman system (532 nm, 100× objective lens).
The time-resolved pump-probe profiles of graphene films were obtained using a femtosecond Ti:sapphire laser system (Spectra Physics). The laser pulses were generated from a mode-locked Ti:sapphire oscillator seeded regenerative amplifier with a pulse energy of $2 \mathrm{~mJ}$, pulse width of $100 \mathrm{fs}$ at $800 \mathrm{~nm}$ and a repetition rate of $1 \mathrm{kHz}$. The $800 \mathrm{~nm}$ laser beam was split into two portions. The larger portion of the beam was directed through a $\beta-\mathrm{Ba}_{2} \mathrm{O}_{4}(\mathrm{BBO})$ crystal to generate the $400 \mathrm{~nm}$ pump beam by frequency-doubling. A small portion of the $800 \mathrm{~nm}$ pulse was used to generate a white light continuum probe beam in a $1 \mathrm{~mm}$ sapphire plate. The signal and reference beams were detected by photodiodes that were connected to lock-in amplifiers and the computer. The pump beam was focused onto the film with a beam size of $200 \mu \mathrm{m}$ and overlapped the smaller-diameter $(100 \mu \mathrm{m})$ probe beam. The delay between the pump and probe pulses was varied by a computer-controlled translation stage. The pump beam was modulated by an optical chopper at a frequency of $500 \mathrm{~Hz}$.

For the power-dependent nonlinear absorption measurements, a stable and standard soliton modelocked fiber laser working at $1550 \mathrm{~nm}$ with output pulse width of $\sim 1$ ps and repetition rate of $\sim 5 \mathrm{MHz}$ was used as input seed pulse, similar to our previous work $[7,11]$. The measurements started at a very low input power of $\sim-40 \mathrm{dBm}$ (i.e., $100 \mathrm{nW}$ ). After the seed pulses passed through monolayer graphene, an output power of $\sim-45 \mathrm{dBm}(31.6 \mathrm{nW})$ was observed. When we gradually increased the input power to $\sim-14 \mathrm{dBm}(0.0398 \mathrm{~mW})$, the output power was very similar, with about $0.15 \mathrm{~dB}$ transmission loss due to saturation. For graphene-based fiber lasers, we used the standard fiber-optic components such as wavelength division multiplexer (WDM), polarization controller (PC), coupler, optical isolator, erbium-doped fiber (EDF), and single mode fiber (SMF). The fiber laser had a ring cavity which consisted of a piece of $6.4 \mathrm{~m}$ EDF with a group velocity dispersion (GVD) parameter of $10\left(\mathrm{ps} \cdot \mathrm{nm}^{-1}\right) \cdot \mathrm{km}^{-1}$, and a total length of $72.2 \mathrm{~m} \mathrm{SMF}$ with GVD parameter of $18\left(\mathrm{ps} \cdot \mathrm{nm}^{-1}\right) \cdot \mathrm{km}^{-1}$. Polarization-independent isolators were used to force the unidirectional operation of the ring, and an intracavity PC was used to optimize the cavity birefringence. The laser was pumped by a high power fiber Raman laser of wavelength $1480 \mathrm{~nm}$. 


\section{Results and discussion}

\subsection{Characterization of monolayer graphene}

The monolayer graphene was synthesized by the CVD approach on a Cu substrate [15], in which uniform, high quality monolayer graphene was obtained through the catalytic decomposition of $\mathrm{CH}_{4}$ on the $\mathrm{Cu}$ surface [17]. Multilayer graphene (1-4 layers) grown on $\mathrm{Ni}$ was also studied for comparison [7]. The graphene film $(5 \mathrm{~mm} \times 5 \mathrm{~mm}$ ) was isolated by etching the $\mathrm{Cu}$ (or Ni) substrate and then it was transferred onto the cross-section of an optical fiber for further studies.

To understand the influences of defects, functional groups and number of layers on the nonlinear optical properties, the following samples were specially prepared for investigation: Monolayer graphene, monolayer graphene covalently bonded to functional groups on its basal plane by diazonium functionalization, monolayer graphene with wrinkles, as well as 1-4 layer and 4-8 layer graphene grown on Ni. Raman spectroscopy was used to evaluate the quality and uniformity of graphene in the fiber core area, as shown in Fig. 1. The location of the $\sim 8 \mu \mathrm{m}$ fiber core was determined by the Raman signal in the $200-600 \mathrm{~cm}^{-1}$ range, which originates from the crystalline $\mathrm{SiO}_{2}$ core, as described elsewhere [7]. Figure 1(a) shows that a monolayer graphene film covers the fiber core area quite homogenously. The corresponding Raman spectrum in Fig. 1(e) reveals that the Raman 2D band is much stronger than the $G$ band with a $2 D / G$ intensity ratio of 2.4 and the $2 \mathrm{D}$ band can be fitted well by a single Lorentzian curve with a narrow full-width at half-maximum (FWHM) of $28 \mathrm{~cm}^{-1}$. The Raman D band, associated with defects [18], is hardly observed. These observations indicate that the as-produced graphene has very high crystalline quality. The atomic force microscopy (AFM) image further confirms the monolayer thickness $(\sim 0.7 \mathrm{~nm})$ of the graphene (see Fig. S-1 in the Electronic Supplementary Material (ESM)). Figure 1(b) reveals that the functionalized monolayer graphene is generally uniform, except for the presence of several wrinkles on the edge area of the fiber core. The corresponding Raman spectrum (Fig. 1(e)) exhibits characteristic peaks of both graphene ( $\mathrm{G}$ and 2D bands) and 4-nitrophenyl groups in the $1000-1500 \mathrm{~cm}^{-1}$ region,

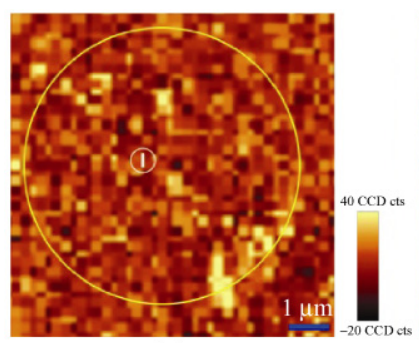

(a)

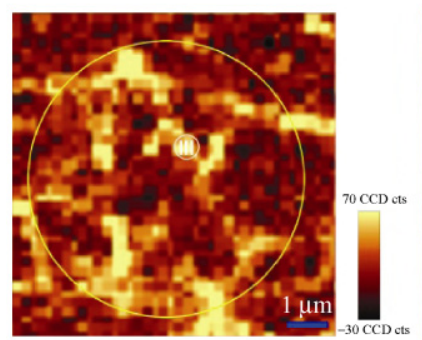

(c)

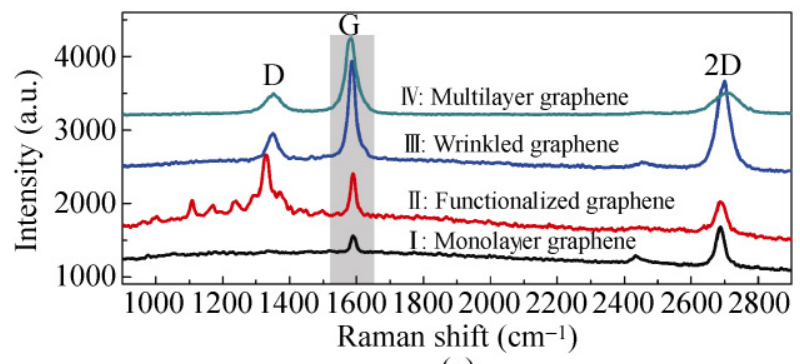

(e)

Figure 1 Micro-Raman characterization of monolayer and multilayer graphene transferred onto optical fibers. Integrated intensity maps of the $\mathrm{G}$ band $\left(1520-1650 \mathrm{~cm}^{-1}\right)$ of (a) monolayer graphene, (b) functionalized monolayer graphene, (c) wrinkled monolayer graphene, and (d) multilayer graphene. The yellow circles indicate the location of the fiber core area of each sample. (e) Raman spectra from spots marked with the corresponding number in (a)-(d)

which suggests functionalization via covalent bonds has been successfully achieved. Figure 1(c) shows that the graphene surface contains many wrinkle-like defects, which are responsible for the strong $\mathrm{D}$ band [19] in the corresponding Raman spectrum in Fig. 1(e). The multilayer graphene (grown on $\mathrm{Ni}$ ) is not uniform in thickness, as shown in Fig. 1(d). The relatively broad 2D band in its Raman spectrum can be fitted by several Gaussian or Lorentzian peaks, which is characteristic of its few-layer thickness.

\subsection{Nonlinear optical properties}

The saturable absorption of the graphene samples was investigated and the results are shown in Fig. 2.

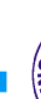

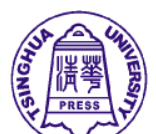

算 Springer 
Figure 2(a) shows the nonlinear absorption properties (in units of $\mathrm{dB}$ ) of monolayer graphene with different surface states. First, it can be seen that the absorption of all the samples decreases sharply at a particular threshold input intensity and becomes nearly constant at higher intensity. This corresponds to typical saturable absorption behavior. It is noteworthy that pristine monolayer graphene (the sample in Fig. 1(a)) only caused $0.14 \mathrm{~dB}$ transmission loss after its absorption was saturated, in contrast to more than $5 \mathrm{~dB}$ initial optical loss with 1 ps laser pulses at lower incident intensity. Notwithstanding the insertion loss of the fiber pigtail $(\sim 2.3 \mathrm{~dB})$, this result reveals that graphene becomes highly transparent after it is saturated. The presence of wrinkles (the sample in Fig. 1(c)) in graphene resulted in slightly higher transmission loss after saturation (inset of Fig. 2(a)), which may originate from nonsaturable optical loss. It was also found that the transmission loss in the saturation regime decreased slightly after the sample was covalently functionalized (the sample in Fig. 1(b)), which might result from $p$-type doping by the 4-nitrophenyl groups.

For the purpose of comparison, the nonlinear saturable absorption of few-layer graphene was also measured, as shown in Fig. 2(b). Thicker graphene films have larger optical loss in both linear and nonlinear regimes. For 1-4 layer graphene (the sample in Fig. 1(d)), the transmission loss decreased from 5.88 to $1.65 \mathrm{~dB}$ with increasing incident intensity, whereas it decreased from 8.55 to $4.92 \mathrm{~dB}$ for the $4-8$ layer graphene sample. The performance of pristine monolayer graphene contrasts sharply with these because the transmission loss decreased dramatically from 5.10 to $0.14 \mathrm{~dB}$ after saturation. These results point to higher nonsaturable loss caused by the nonuniformity and scattering of thicker graphene films. We should also note that the whole nonlinear absorption curves shift in the direction of higher input intensity for thicker films, which reflects the fact that higher input power is needed to saturate all the graphene layers in the longer optical absorption path. The strong scattering also contributes to this shift as some of the input power is wasted.

The nonlinear absorption data collected above were converted into percentage transmission in order to clearly compare the two components of nonlinear

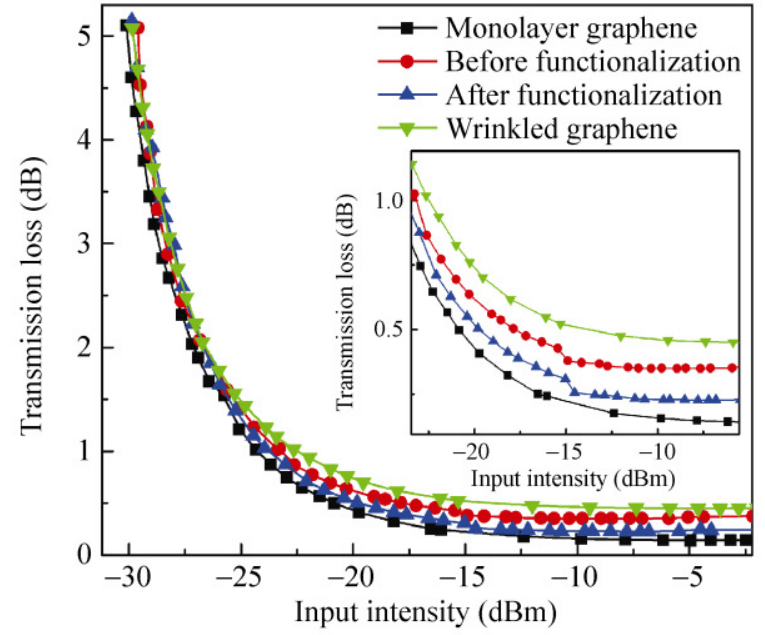

(a)

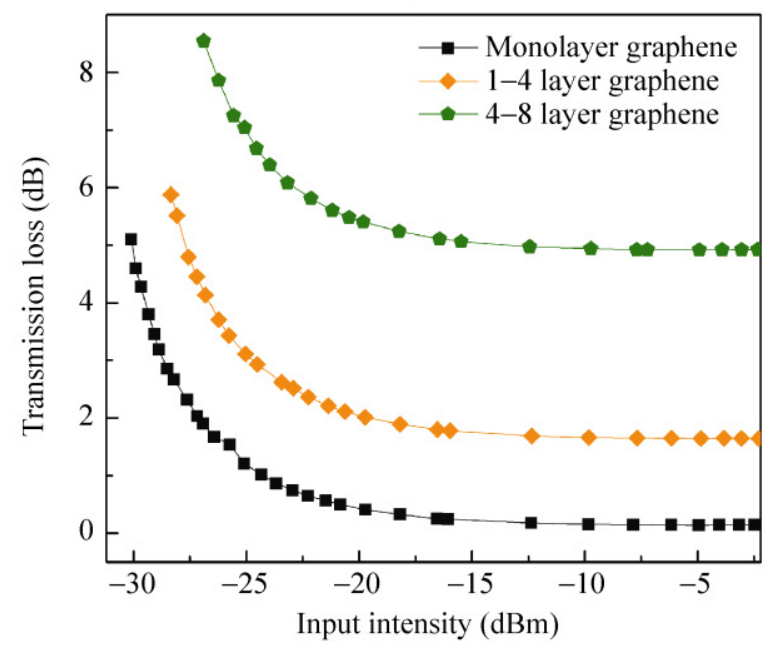

(b)

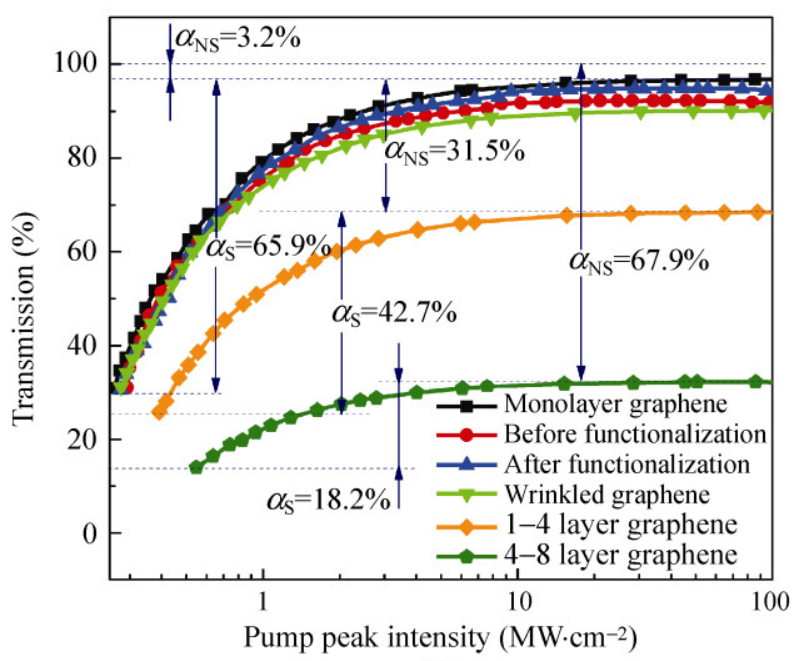

(c)

Figure 2 Power-dependent nonlinear absorption properties. (a) Monolayer graphene samples with different surface states. The inset shows enlarged plots. (b) Comparison of monolayer graphene with multilayer graphene. (c) Power-dependent percentage transmission 
absorption: Saturable absorption and nonsaturable absorption, as shown in Fig. 2(c). The saturable absorption is also termed modulation depth, and is very important in evaluating the pulse shaping abilities of saturable absorbers. The saturation intensity, i.e., the optical intensity required in a steady state to reduce the absorption to half of its unbleached value, can be estimated by fitting the nonlinear absorption curves using the formula

$$
\alpha(I)=\frac{\alpha_{\mathrm{S}}}{1+I / I_{\mathrm{S}}}+\alpha_{\mathrm{NS}}
$$

where $\alpha_{\mathrm{S}}$ and $\alpha_{\mathrm{NS}}$ are the saturable and nonsaturable absorption, and $I_{S}$ is the saturation intensity [11]. These saturable absorption properties are summarized in Table 1.

The remarkable result is that pristine monolayer graphene has a modulation depth of $65.9 \%$, which is much larger than those reported in previous modelocking studies on graphene $[7,8,10,11]$, as well as those of any other known saturable absorbers [20,21]. It can be concluded that both surface defects (wrinkles) and thickness result in smaller modulation depth due to the increased nonsaturable absorption. After functionalization, the modulation depth was enhanced slightly from $59.7 \%$ to $64.4 \%$. The saturation intensity shows the opposite trend as a function of number of surface defects and thickness. The pristine monolayer graphene has the smallest saturation intensity of 0.53 $\mathrm{MW} \cdot \mathrm{cm}^{-2}$, while the presence of two small wrinkles on the surface (shown in Fig. 1(b)) leads to a $4 \%$ increase in the saturation intensity. The 1-4 layer graphene film has a saturation intensity of $0.77 \mathrm{MW} \cdot \mathrm{cm}^{-2}$, which agrees well with previous work [7]. Scaling with thickness, the 4-8 layer graphene needs much more incident light power to reach saturation, with a saturation intensity of $1.09 \mathrm{MW} \cdot \mathrm{cm}^{-2}$. We suggest that the defect-induced nonsaturable loss and scattering play a dominant role in determining the saturation intensity.

\subsection{Carrier dynamics}

To understand the saturation mechanism, especially with respect to the dynamics of state filling by the photocarriers which lead to Pauli blocking, pumpprobe experiments were carried out. Figures 3(a) and 3(b) show the measured transmittivity transients of pristine monolayer graphene and 1-4 layer graphene, which was pumped at $400 \mathrm{~nm}$ and probed at $750 \mathrm{~nm}$. The data can be well fitted using a bi-exponentially decaying function, $\Delta T(t) / T=A_{1} \exp \left(-t / \tau_{1}\right)+A_{2} \exp \left(-t / \tau_{2}\right)$, convoluted with the cross-correlation of the pump and probe pulses. The carrier relaxation time comprises a fast time constant $\left(\tau_{1}\right)$ in the 100-150 fs range, resulting from carrier-carrier intraband scattering processes, and a slower time constant $\left(\tau_{2}\right)$ in the $405-570 \mathrm{fs}$ range, corresponding to carrier-phonon intraband scattering and electron-hole recombination (Auger scattering). The initial fast relaxation time $\tau_{1}$ of monolayer graphene is around $100 \mathrm{fs}$, which agrees with previous reports for graphene [3, 22-24]. However, we should note that the faster time $\tau_{1}$ is of the order of the pulse width and is, therefore, not accurately resolved. Compared with the theoretical prediction by Rana et al. [25], the electron-hole recombination rate $\left(\tau_{2}\right)$ is quite

Table 1 Saturable absorption properties of monolayer and multilayer graphene

\begin{tabular}{|c|c|c|c|c|c|}
\hline \multirow{2}{*}{$\begin{array}{l}\text { Sample } \\
\text { number }\end{array}$} & \multicolumn{2}{|c|}{ Transmission loss } & \multirow{2}{*}{$\begin{array}{l}\text { Saturation intensity } \\
\quad\left(\mathrm{MW} \cdot \mathrm{cm}^{-2}\right)\end{array}$} & \multicolumn{2}{|c|}{ Nonlinear absorption } \\
\hline & Starting point $(\mathrm{dB})$ & After saturation (dB) & & $\alpha_{\mathrm{S}}(\%)$ & $\alpha_{\mathrm{NS}}(\%)$ \\
\hline 1 & 5.10 & 0.14 & 0.53 & 65.88 & 3.24 \\
\hline 2 & 5.08 & 0.35 & 0.55 & 59.65 & 9.30 \\
\hline 3 & 5.15 & 0.23 & 0.57 & 64.36 & 5.09 \\
\hline 4 & 5.08 & 0.45 & 0.54 & 59.02 & 9.90 \\
\hline 5 & 5.88 & 1.65 & 0.77 & 42.69 & 31.48 \\
\hline 6 & 8.55 & 4.92 & 1.09 & 18.17 & 67.85 \\
\hline
\end{tabular}

1: Monolayer graphene. 2: Monolayer graphene with two wrinkles before functionalization. 3: Monolayer graphene with two wrinkles after functionalization. 4: Monolayer graphene with many wrinkles. 5: 1-4 layer graphene grown on Ni. 6: 4-8 layer graphene grown on $\mathrm{Ni}$ 
fast $(<1 \mathrm{ps})$ in our experiment. This can be explained by the dependence of charge screening and Coulomb scattering on the dielectric constant of the surrounding media. Contrary to Dawlaty's observation that larger crystal disorder results in shorter relaxation time $\left(\tau_{2}\right)$ in epitaxial graphene [22], we observed that the 1-4 layer graphene has longer relaxation time (both $\tau_{1}$ and $\tau_{2}$ ) than monolayer graphene even though it has a stronger defect-related D peak (this might also originate from the edges of inhomogeneously stacked graphene layers) in its Raman spectrum. This trend is however consistent with Newson's observations of the layer dependence of carrier relaxation decay time [23].

We can estimate the saturated carrier density using a simplified rate equation which involves long-pulse excitation [7]

$$
N=\frac{\alpha I \tau}{\hbar \omega}
$$

where $\alpha$ is the absorption coefficient, $I$ is the incident intensity, $\tau$ is the carrier recombination time, $\hbar$ is the reduced Planck's constant and $\omega$ is the frequency of light. Here $\hbar \omega=0.8 \mathrm{eV}$, as the wavelength of the pump laser for the saturable absorption measurements was $1550 \mathrm{~nm}$. For monolayer graphene, if we assume that $\tau \approx \tau_{2}=405 \mathrm{fs}$, and $I_{\mathrm{s}}=0.53 \mathrm{MW} \cdot \mathrm{cm}^{-2}$, this gives a saturated carrier density $N_{\mathrm{s}}=1.06 \times 10^{13} \mathrm{~cm}^{-2}$. According to the relationship between Fermi energy and carrier density at low temperature [26, 27],

$$
E_{\mathrm{F}}(n)=\hbar\left|v_{\mathrm{F}}\right| \sqrt{\pi n}
$$

where $\left|v_{\mathrm{F}}\right|$ is the Fermi velocity and $n$ is the carrier density in units of $\mathrm{cm}^{-2}$, this amount of carriers can fill the cone-shaped conduction band of graphene up to $0.43 \mathrm{eV}$ (this value is an over-estimate as the spread of carrier distribution at finite temperature is ignored), which is very close to the electron energy excited by our pump laser, which is $\hbar \omega / 2=0.4 \mathrm{eV}$. Therefore, we can conclude that all the electron states below $\hbar \omega / 2$ have been occupied by pumped electrons, prohibiting the optical transitions and further creation of electron-hole pairs due to Pauli blocking, as schematically illustrated by the inset in Fig. 3(c).

Energy-dependent relaxation time measurements were carried out by varying the probe laser wavelength, as shown in Fig. 3(c), in which the trace for graphite extracted from Ref. [14] is also included for comparison. It was found that the measured lifetime of graphene is longer than graphite since the faster relaxation time $\tau_{1}$ is not resolvable, limited by the pulse width used in our measurements. The quasiparticle lifetime

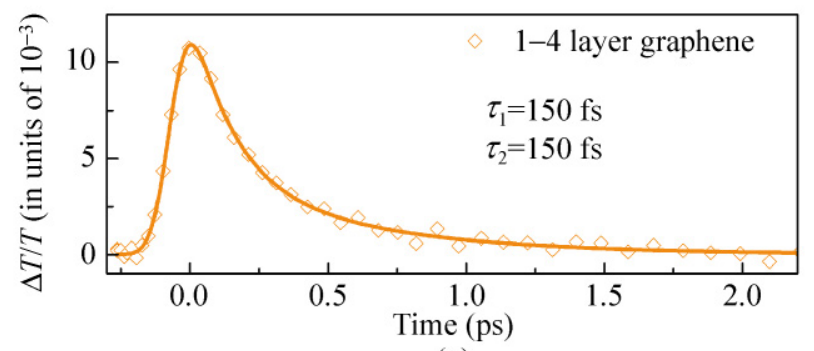

(a)

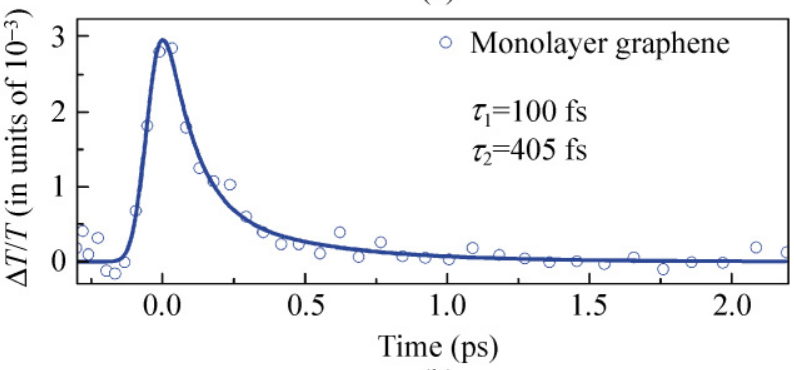

(b)

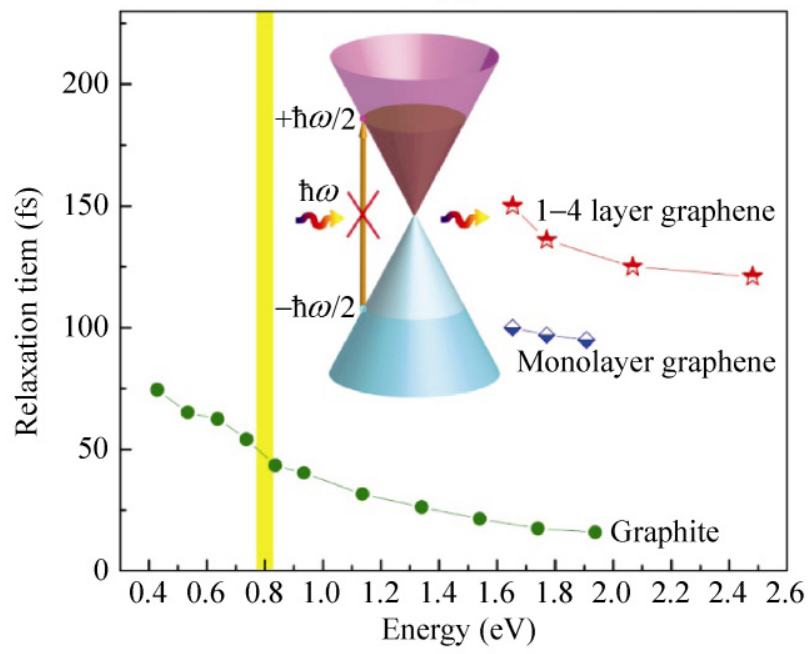

(c)

Figure 3 Measured transmittivity transients of graphene samples. (a) 1-4 layer graphene. (b) Monolayer graphene. The samples were pumped at $400 \mathrm{~nm}$ and probed at $750 \mathrm{~nm}$ with $20 \mathrm{~mW}$ incident laser power. (c) Energy-dependent relaxation time $\left(\tau_{1}\right)$ measurements pumped at $400 \mathrm{~nm}$ and probed at 500,600,700, and $750 \mathrm{~nm}$. The green solid dot curve for graphite was adapted from Ref. [14] for comparison (Copyright 1996, The American Physical Society). The yellow region indicates the operating window $(\sim 1550 \mathrm{~nm}, 0.8 \mathrm{eV})$ for the telecommunication C-band. Inset: A schematic illustration of saturable absorption in graphene due to Pauli blocking 
in graphite has been theoretically and experimentally demonstrated to be inversely proportional to the quasiparticle energy above the Fermi level [14, 28], due to the linear dispersion around the Fermi level, although there have been some reports of deviations between 1.1 and $1.5 \mathrm{eV}[29,30]$. The key message of Fig. 3(c) is that it suggests that monolayer and multilayer graphene follow the general trend for graphite, i.e., lower excitation energy correlates with longer carrier lifetime. At the working wavelength of the telecommunication C-band $(\sim 1550 \mathrm{~nm})$, the lifetime should be longer than $100 \mathrm{fs}$ in monolayer graphene. Similar to conventional semiconductor absorbers, the slower relaxation time constant $\left(\tau_{2}\right)$ results in reduced saturation intensity for part of the absorption and hence facilitates self-starting mode-locking. In contrast, the faster relaxation time $\left(\tau_{1}\right)$ constant is more effective in shaping ultra-short laser pulses [20, 21]. Accordingly, monolayer graphene should have better pulse shaping performance than multilayer graphene, due to the shorter relaxation time $\left(\tau_{1}\right)$ of the order of $\sim 100 \mathrm{fs}$.

\subsection{Pulse shaping ability}

To evaluate the pulse shaping ability of graphene and correlate this with its carrier relaxation time $\left(\tau_{1}\right)$, we carried out pulse dynamics simulations. As mentioned above, the absorption in graphene can be saturated under strong excitation due to the depletion of final states (i.e., Pauli blocking). Within $100 \mathrm{fs}$, the excited carriers in each band thermalize, which leads to partial recovery of the absorption. Such carrier dynamics in graphene influences the intracavity mode-locked pulse behaviors, i.e., longer pulses transmitted through graphene would encounter further pulse shaping (the lower intensity wing will be absorbed), owing to the fast relaxation time of graphene. In order to quantitatively characterize the pulse shaping ability of graphene, comprehensive numerical simulations of the pulse dynamics in the laser cavity were carried out based on the coupled Ginzburg-Landau equations (for details see the ESM and Refs. [31, 32]). The advantage of such simulations is that one can get an insight into the pulse evolution in the cavity, especially the pulse shaping caused by each of the cavity components, which is not easily visualized by experiment.
Under stable mode-locking operation, the pulse durations before and after passing through the graphene were compared, as shown in Fig. 4. Accordingly, the ratio of pulse width variations was adopted to feature graphene's pulse shaping ability (see the inset of Fig. 4(b)). Figure 4(a) shows the calculated soliton pulse before and after passing through graphene, obtained with relaxation time $=100 \mathrm{fs}$, gain $=15.5 \mathrm{~dB}$ and a very weak cavity birefringence of $L / L_{b}=0.1$. Before passing through graphene, the pulse width was about $1.93 \mathrm{ps}$. After passing through graphene an obvious pulse width narrowing was observed, with the pulse width becoming about $1.66 \mathrm{ps}$. Considering that the relaxation time of graphene can vary significantly from sample to sample due to defects, chemical

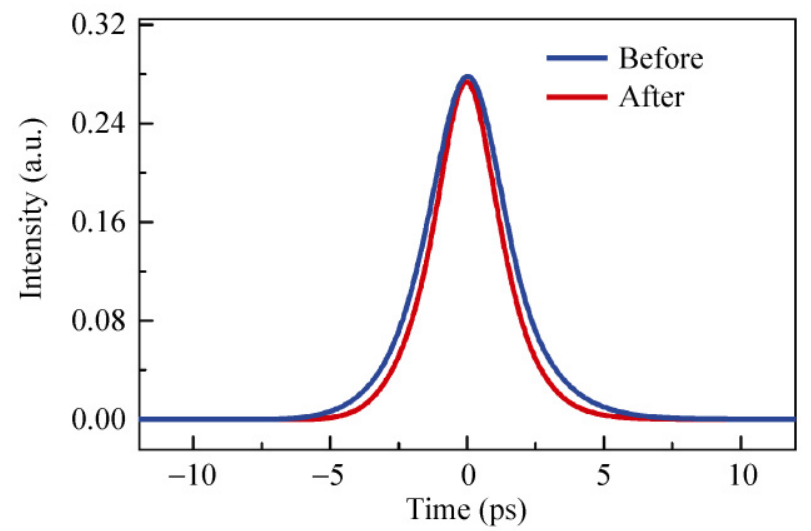

(a)

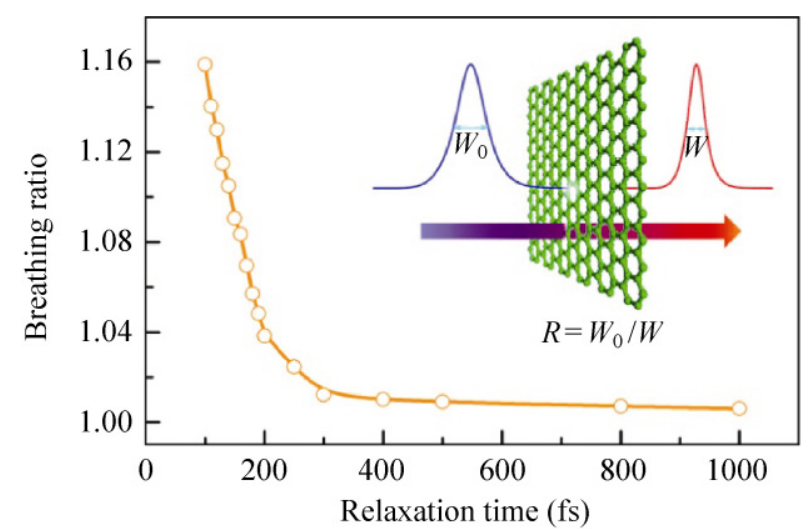

(b)

Figure 4 (a) Calculated soliton pulse duration before and after it passes through graphene. (b) Breathing ratio of graphene as a function of relaxation time. Inset: The pulse shaping by graphene and definition of breathing ratio $R=W_{0} / W$, where $W_{0}$ is the pulse width before passing through graphene and $W$ is the pulse width after passing through graphene 
functionalization and nonuniform thickness, we calculated the pulse width narrowing obtained using the same laser parameters but with different saturable relaxation times. Figure $4(\mathrm{~b})$ shows the values of the simulated breathing ratio as a function of relaxation time. It shows that in a mode-locked fiber laser, the pulse shaping ability of graphene becomes less significant once its relaxation time becomes slower. This indicates that graphene having faster relaxation times possesses stronger pulse shaping ability, which points to the potential optoelectronic application of monolayer graphene as pulse shapers.

\subsection{Mode-locked laser}

Finally, monolayer graphene was incorporated into a ring-configuration fiber laser cavity, as shown in Fig. 5(a). Figures 5(b)-5(f) show representative mode-locking characteristics of monolayer graphene. Figure 5(b) shows the oscilloscope trace of the laser output where a pulse repeats every $\sim 393.3 \mathrm{~ns}$, matching exactly with the cavity length of $\sim 78.6 \mathrm{~m}$. In a laser cavity, the boundary condition causes light to be emitted at discrete frequencies, known as modes. When a saturable absorber material such as graphene is used to "mode-lock" these modes, the phases of all the modes become synchronized, and a stable and intense single bright pulse is formed (Fig. 5(b)). The corresponding mode-locking spectrum in Fig. 5(c) has a central wavelength located at $1561 \mathrm{~nm}$ and a $3 \mathrm{~dB}$ bandwidth of more than $5 \mathrm{~nm}$. Experimentally, once the pump power is raised above a threshold $(\sim 8 \mathrm{~mW})$, we can always observe the mode-locking spectra with clear and symmetric Kelly sidebands, as shown in Fig. 5(c), which are caused by constructive interference between the soliton and dispersive waves emitted from the soliton. In such a soliton mode-locking regime, the function of the saturable absorber (i.e., graphene) is to initiate pulse shaping and stabilize the pulse. Subsequently, other laser cavity properties such as dispersion will play more important roles in determining the pulse parameters [33]. The resulting soliton displays nonlinear Schrödinger equation soliton features [32]; in this case the total cavity dispersion is anomalous and the intrinsic soliton shaping mechanism arises from the interplay between the anomalous cavity dispersion and nonlinear optical Kerr effect of the fiber.

The radio frequency (RF) spectrum of the modelocked pulses was also measured, as shown in Fig. 5(d), with a signal-to-noise ratio of $60 \mathrm{~dB}\left(10^{6}\right.$ contrast). This indicates that the laser is operating in an excellent CW mode-locking regime. An output power up to $3 \mathrm{~mW}$ was recorded with a slope efficiency of $2.2 \%$ (Fig. 5(e)). This result is comparable to that for singlewalled carbon nanotube (SWNT) mode-locked fiber lasers $[34,35]$ but it could be further improved by laser cavity design, i.e., larger cavity output ratio and weaker splicing loss. The pump threshold for selfstarting of the mode-locking ranged from 8 to $40 \mathrm{~mW}$, similar to the values for multilayer graphene [7]. Since a monolayer graphene saturable absorber has relatively lower insertion loss than multilayer graphene, in principle, lower threshold pump energy is required to initiate the mode-locking performance. However, modulation depth and recovery time also play important roles in determining the mode-locking ability of saturable absorbers. Due to the fact that monolayer graphene has extremely large modulation depth together with fast decay time, higher pump energy is desirable to stabilize the mode-locking operation. Consequently, we do not observe a significant change in the self-starting threshold of mode-locking for monolayer graphene. The autocorrelation (AC) trace of the mode-locked pulses is shown in Fig. 5(f), and is well fitted by a sech ${ }^{2}$ profile with full-width at halfmaximum (FWHM) of 1.90 ps. The real pulse width is then obtained by multiplying the AC trace width with the de-correlation factor, which is 0.648 for the $\mathrm{sech}^{2}$ pulses. This gives a pulse width of $1.23 \mathrm{ps}$, which is comparable with those of the pulses obtained in multilayer graphene [7] or SWNT mode-locked fiber lasers [36].

In a series of comparative mode-locking studies using carbon nanotube saturable absorbers, we observed that pulse splitting is more likely to occur in the same laser cavity. In terms of laser performance, the larger modulation depth of monolayer graphene ensures long term stability of the generated laser pulses by avoiding pulse splitting. On the other hand, the small scattering loss favors the generation of high energy 


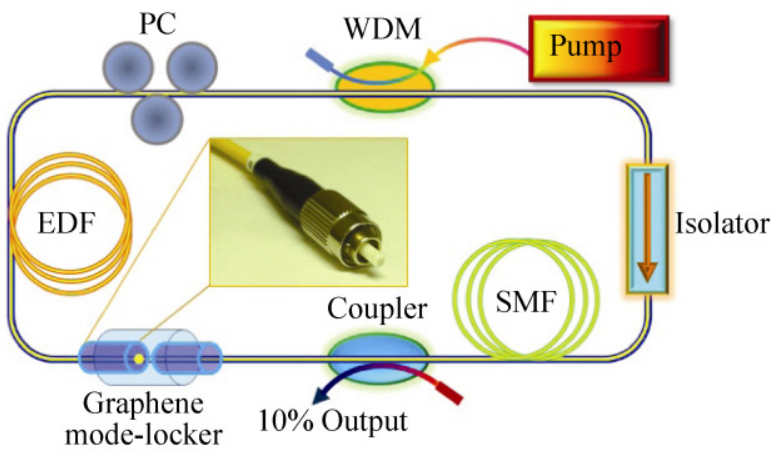

(a)

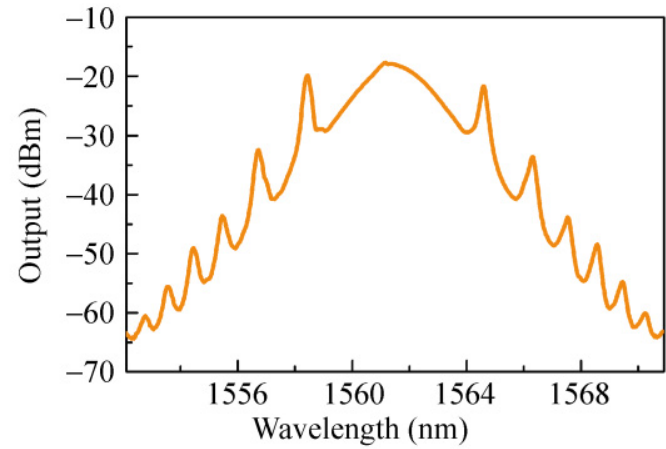

(c)

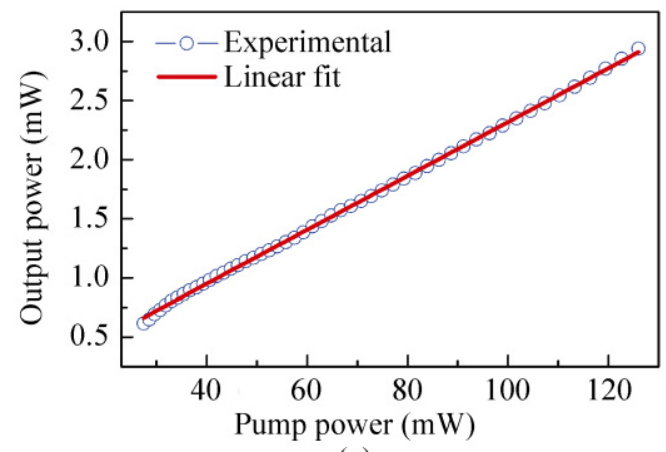

(e)

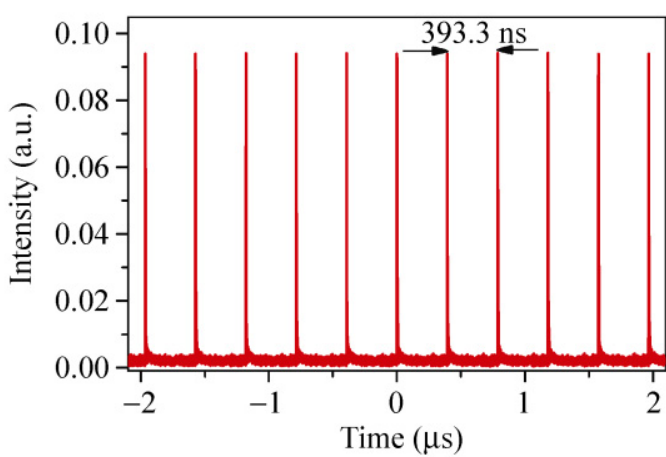

(b)

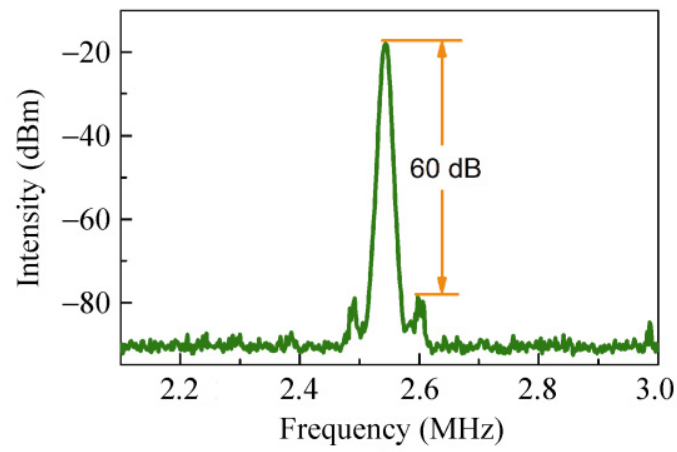

(d)

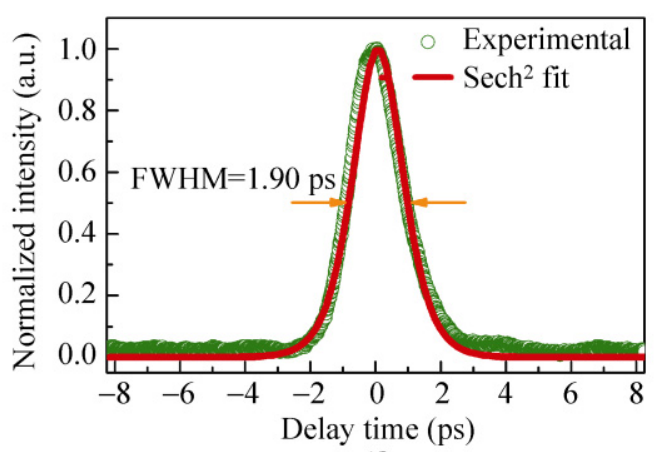

(f)

Figure 5 Mode-locking characteristics of monolayer graphene. (a) Schematic view of the laser cavity (WDM: Wavelength division multiplexer; PC: Polarization controller; EDF: Erbium-doped fiber; SMF: Single mode fiber). Inset: Photograph of the fiber pigtail with a graphene film coating on the end face. (b) Output pulse train. (c) Typical output spectrum centered at $1561 \mathrm{~nm}$. (d) Radio frequency spectrum. (e) Output power as a function of pump power. (f) AC trace and $\operatorname{sech}^{2}$ fitting curve; a 1.23 ps pulse is obtained

pulses with the same laser cavity design [37]. According to our preliminary experimental results, high energy mode-locking up to $20 \mathrm{~nJ}$ was obtained by using monolayer graphene as a saturable absorber in a normal dispersion laser cavity, which is much higher than that obtained with multilayer graphene $(7.3 \mathrm{~nJ})$ in a similar cavity [8]. Furthermore, the mode-locking performance does not degrade over a few weeks. This aspect will be further investigated.

\section{Conclusions}

We have comparatively evaluated the saturable absorption properties of monolayer graphene, multilayer graphene, and defective graphene in order to gain an insight into how layer thickness and presence of defects affect mode-locking performance. In terms of saturation threshold energy, modulation depth and pulse shaping ability, monolayer graphene shows a 
superior performance to those of thicker or defective graphene layers. Pulse dynamic simulation reveals that the pulse shaping ability is correlated with the ultrafast relaxation time of monolayer graphene. Monolayer graphene can be saturated at a remarkably low excitation intensity of $0.53 \mathrm{MW} \cdot \mathrm{cm}^{-2}$ and its modulation depth of $65.9 \%$ is possibly the largest of all known saturable absorbers reported so far. We further demonstrated that picosecond ultrafast laser pulses (1.23 ps) can be generated using monolayer graphene as a saturable absorber.

\section{Acknowledgements}

Q. Bao and H. Zhang contributed equally to the project. The authors thank the National Research Foundation Competitive Research Program (NRF-CRP) grant "Graphene Related Materials and Devices" No. R-143000-360-281.

Electronic Supplementary Material: Supplementary material (AFM image of monolayer graphene and details of pulse dynamics simulations) is available in the online version of this article at http://dx.doi.org/ 10.1007/s12274-010-0082-9.

\section{References}

[1] Novoselov, K. S.; Geim, A. K.; Morozov, S. V.; Jiang, D.; Zhang, Y.; Dubonos, S. V.; Grigorieva, I. V.; Firsov, A. A. Electric field effect in atomically thin carbon films. Science 2004, 306, 666-669.

[2] Novoselov, K. S.; Geim, A. K.; Morozov, S. V.; Jiang, D.; Katsnelson, M. I.; Grigorieva, I. V.; Dubonos, S. V.; Firsov, A. A. Two-dimensional gas of massless Dirac fermions in graphene. Nature 2005, 438, 197-200.

[3] George, P. A.; Strait, J.; Dawlaty, J.; Shivaraman, S.; Chandrashekhar, M.; Rana, F.; Spencer, M. G. Ultrafast optical-pump terahertz-probe spectroscopy of the carrier relaxation and recombination dynamics in epitaxial graphene. Nano Lett. 2008, 8, 4248-4251.

[4] Sun, D.; Wu, Z. K.; Divin, C.; Li, X. B.; Berger, C.; de Heer, W. A.; First, P. N.; Norris, T. B. Ultrafast relaxation of excited Dirac fermions in epitaxial graphene using optical differential transmission spectroscopy. Phys. Rev. Lett. 2008, $101,157402$.

[5] Kuzmenko, A. B.; van Heumen, E.; Carbone, F.; van der
Marel, D. Universal optical conductance of graphite. Phys. Rev. Lett. 2008, 100, 117401.

[6] Nair, R. R.; Blake, P.; Grigorenko, A. N.; Novoselov, K. S.; Booth, T. J.; Stauber, T.; Peres, N. M. R.; Geim, A. K. Fine structure constant defines visual transparency of graphene. Science 2008, 320, 1308.

[7] Bao, Q.; Zhang, H.; Wang, Y.; Ni, Z.; Yan, Y.; Shen, Z. X.; Loh, K. P.; Tang, D. Y. Atomic-layer graphene as a saturable absorber for ultrafast pulsed lasers. Adv. Funct. Mater. 2009, 19, 3077-3083.

[8] Zhang, H.; Tang, D. Y.; Zhao, L. M.; Bao, Q.; Loh, K. P. Large energy mode-locking of an erbium-doped fiber laser with atomic layer graphene. Opt. Express 2009, 17, 1763017635.

[9] Zhang, H.; Tang, D.; Knize, R. J.; Zhao, L.; Bao, Q.; Loh, K. P. Graphene mode-locked, wavelength-tunable, dissipative soliton fiber laser. Appl. Phys. Lett. 2010, 96, 111112.

[10] Zhang, H.; Bao, Q.; Tang, D. Y.; Zhao, L.; Loh, K. P. Large energy soliton erbium-doped fiber laser with a graphenepolymer composite mode-locker. Appl. Phys. Lett. 2009, 95, 141103.

[11] Bao, Q.; Zhang, H.; Yang, J. X.; Wang, S.; Tang, D. Y.; Jose, R.; Ramakrishna, S.; Lim, C. T.; Loh, K. P. Graphenepolymer nanofiber membrane for ultrafast photonics. $A d v$. Funct. Mater. 2010, 20, 782-791.

[12] Song, Y. W.; Jang, S. Y.; Han, W. S.; Bae, M. K. Graphene mode-lockers for fiber lasers functioned with evanescent field interaction. Appl. Phys. Lett. 2010, 96, 051122.

[13] Sun, Z.; Hasan, T.; Torrisi, F.; Popa, D.; Privitera, G.; Wang, F.; Bonaccorso, F.; Basko, D. M.; Ferrari, A. C. Graphene mode-locked ultrafast laser. ACS Nano 2010, 4, 803-810.

[14] Xu, S.; Cao, J.; Miller, C. C.; Mantell, D. A.; Miller, R. J. D.; Gao, Y. Energy dependence of electron lifetime in graphite observed with femtosecond photoemission spectroscopy. Phys. Rev. Lett. 1996, 76, 483-486.

[15] Li, X.; Cai, W.; An, J.; Kim, S.; Nah, J.; Yang, D.; Piner, R.; Velamakanni, A.; Jung, I.; Tutuc, E. Large-area synthesis of high-quality and uniform graphene films on copper foils. Science 2009, 324, 1312-1314.

[16] Kim, K. S.; Zhao, Y.; Jang, H.; Lee, S. Y.; Kim, J. M.; Ahn, J. H.; Kim, P.; Choi, J. Y.; Hong, B. H. Large-scale pattern growth of graphene films for stretchable transparent electrodes. Nature 2009, 457, 706-710.

[17] Li, X.; Cai, W.; Colombo, L.; Ruoff, R. S. Evolution of graphene growth on $\mathrm{Ni}$ and $\mathrm{Cu}$ by carbon isotope labeling. Nano Lett. 2009, 9, 4268-4272.

[18] Ferrari, A.; Meyer, J.; Scardaci, V.; Casiraghi, C.; Lazzeri, M.; Mauri, F.; Piscanec, S.; Jiang, D.; Novoselov, K.; Roth, 
S. Raman spectrum of graphene and graphene layers. Phys. Rev. Lett. 2006, 97, 187401.

[19] Casiraghi, C.; Hartschuh, A.; Qian, H.; Piscanec, S.; Georgi, C.; Fasoli, A.; Novoselov, K.; Basko, D.; Ferrari, A. Raman spectroscopy of graphene edges. Nano Lett. 2009, 9, 1433 1441.

[20] Keller, U.; Weingarten, K. J.; Kärtner, F. X.; Kopf, D.; Braun, B.; Jung, I. D.; Fluck, R.; Hönninger, C.; Matuschek, N.; der Au, J. A. Semiconductor saturable absorber mirrors (SESAM's) for femtosecond to nanosecond pulse generation in solid-state lasers. IEEE J. Sel. Top. Quantum Electron. 1996, 2, 435-453.

[21] Keller, U. Recent developments in compact ultrafast lasers. Nature 2003, 424, 831-838.

[22] Dawlaty, J. M.; Shivaraman, S.; Chandrashekhar, M.; Rana, F.; Spencer, M. G. Measurement of ultrafast carrier dynamics in epitaxial graphene. Appl. Phys. Lett. 2008, 92, 042116.

[23] Newson, R. W.; Dean, J.; Schmidt, B.; van Driel, H. M. Ultrafast carrier kinetics in exfoliated graphene and thin graphite films. Opt. Express 2009, 17, 2326-2333.

[24] Kumar, S.; Anija, M.; Kamaraju, N.; Vasu, K. S.; Subrahmanyam, K. S.; Sood, A. K.; Rao, C. N. R. Femtosecond carrier dynamics and saturable absorption in graphene suspensions. Appl. Phys. Lett. 2009, 95, 191911.

[25] Rana, F. Electron-hole generation and recombination rates for Coulomb scattering in graphene. Phys. Rev. B 2007, 76, 155431.

[26] Das, A.; Pisana, S.; Chakraborty, B.; Piscanec, S.; Saha, S.; Waghmare, U.; Novoselov, K.; Krishnamurthy, H.; Geim, A.; Ferrari, A. Monitoring dopants by Raman scattering in an electrochemically top-gated graphene transistor. Nat. Nanotechnol. 2008, 3, 210-215.

[27] Yan, J.; Zhang, Y.; Kim, P.; Pinczuk, A. Electric field effect tuning of electron-phonon coupling in graphene. Phys. Rev. Lett. 2007, 98, 166802.

[28] González, J.; Guinea, F.; Vozmediano, M. A. H. Uncon- ventional quasiparticle lifetime in graphite. Phys. Rev. Lett. 1996, 77, 3589-3592.

[29] Spataru, C. D.; Cazalilla, M. A.; Rubio, A.; Benedict, L. X.; Echenique, P. M.; Louie, S. G. Anomalous quasiparticle lifetime in graphite: Band structure effects. Phys. Rev. Lett. 2001, 87, 246405.

[30] Moos, G.; Gahl, C.; Fasel, R.; Wolf, M.; Hertel, T. Anisotropy of quasiparticle lifetimes and the role of disorder in graphite from ultrafast time-resolved photoemission spectroscopy. Phys. Rev. Lett. 2001, 87, 267402.

[31] Zhang, H.; Tang, D. Y.; Zhao, L. M.; Xiang, N. Coherent energy exchange between components of a vector soliton in fiber lasers. Opt. Express 2008, 16, 12618-12623.

[32] Tang, D. Y.; Zhang, H.; Zhao, L. M.; Wu, X. Observation of high-order polarization-locked vector solitons in a fiber laser. Phys. Rev. Lett. 2008, 101, 153904.

[33] Zhang, H.; Tang, D. Y.; Zhao, L. M.; Bao, Q. L.; Loh, K. P.; Lin, B.; Tjin, S. C. Compact graphene mode-locked wavelength-tunable erbium-doped fiber lasers: From all anomalous dispersion to all normal dispersion. Laser Phys. Lett. 2010, 7, 591-596.

[34] Scardaci, V.; Rozhin, A. G.; Tan, P. H.; Wang, F.; White, I. H.; Milne, W. I.; Ferrari, A. C. Carbon nanotubes for ultrafast photonics. Phys. Status Solidi B 2007, 244, 4303-4307.

[35] Scardaci, V.; Sun, Z.; Wang, F.; Rozhin, A.; Hasan, T.; Hennrich, F.; White, I.; Milne, W.; Ferrari, A. Carbon nanotube polycarbonate composites for ultrafast lasers. Adv. Mater. 2008, 20, 4040-4043.

[36] Yamashita, S.; Inoue, Y.; Maruyama, S.; Murakami, Y.; Yaguchi, H.; Jablonski, M.; Set, S. Y. Saturable absorbers incorporating carbon nanotubes directly synthesized onto substrates and fibers and their application to mode-locked fiber lasers. Opt. Lett. 2004, 29, 1581-1583.

[37] Wise, F.; Chong, A.; Renninger, W. High-energy femtosecond fiber lasers based on pulse propagation at normal dispersion. Laser Photonics Rev. 2008, 2, 58-73. 